\title{
The Mass, Normalization and Late Time behavior of the Tachyon Field
}

\author{
A. de la Macorra, U. Filobello \\ Instituto de Física, UNAM, Apdo. Postal 20-364, 01000 México D.F., México \\ G. Germán \\ Centro de Ciencias Físicas, Universidad Nacional Autónoma de México, \\ Apartado Postal 48-3, 62251 Cuernavaca, Morelos, México
}

\begin{abstract}
We study the dynamics of the tachyon field $T$. We derive the mass of the tachyon as the pole of the propagator which does not coincide with the standard mass given in the literature in terms of the second derivative of $V(T)$ or $\log [V(T)]$. We determine the transformation of the tachyon in order to have a canonical scalar field $\phi$. This transformation reduces to the one obtained for small $\dot{T}$ but it is also valid for large values of $\dot{T}$. This is specially interesting for the study of dark energy where $\dot{T} \simeq 1$. We also show that the normalized tachyon field $\phi$ is constrained to the interval $T_{2} \leq T \leq T_{1}$ where $T_{1}, T_{2}$ are zeros of the original potential $V(T)$. This results shows that the field $\phi$ does not know of the unboundedness of $V(T)$, as suggested for bosonic open string tachyons. Finally we study the late time behavior of tachyon field using the L'Hôpital rule.
\end{abstract}

\section{INTRODUCTION}

In recent times a great amount of work has been invested in studying the dynamics of tachyon field [1],2]. This field is motivated from string-brane physics and represents the lowest energy level of an unstable Dp-brane or that of a brane-antibrane system [3]. One of the generic properties of this class of systems is precisely, the existence of the tachyon field $T$. Therefore, the phenomenology of the tachyon is very important in understanding the low energy limit of string-brane models.

We would like to determine some of the physical properties of the tachyon which are important to set up the transition from string-brane theory to an effective low energy 4-D field theory [3]. Since the tachyon field is the last surviving mode of unstable branes, the tachyon reheating or decay processes are specially important in the low energy theory. The usual reheating or decay processes are given in terms of the coupling of the original field to other lighter fields (scalars or fermions) and to its mass. Therefore, the phenomenology of the tachyon depends heavily on its mass.

In the case of Dp-brane systems in superstrings, the potential $V(T)$ has been conjectured 3] to be tachyonic at the origin $T=0$, have a $Z_{2}$ symmetry $T \rightarrow-T$, and have a stable minimum. On the other hand for a bosonic Dp-brane systems, the potential $V(T)$ has been conjectured [3] to be tachyonic at the origin, have a minimum for $T=T_{1}>0$, with $V\left(T_{1}\right)=0$, and be unbounded from below for $T<0$ with $V\left(T_{2}\right)=0$. The study of bosonic tachyons has not been as exhaustive as for the superstring case because it has been argued that the unboundedness of the potential is a problem and because potentials with a minimum at a finite value of $T$ are unstable [4]. Here we will show that the normalized tachyon $\phi(T)$, defined below, does not see the unboundedness of $V(T)$ since the field $\phi(T)$ is constrained in the interval $T_{2} \leq T \leq T_{1}$

In this work we are interested in determining the transformation which renders the tachyon field $T$ into a stan- dard normalized scalar field $\phi$ and derive the effective potential $U(\phi(T))$. The transformation we obtain reduces to the one obtained for small kinetic term $|\dot{T}| \ll 1$ but is also valid for large values of $\dot{T}$. This is specially relevant for the study of dark energy where $\dot{T} \simeq 1$ with $V(T) \simeq 0$.

We study the late time behavior of $T$ and $\phi$ and with the use of the L'Hôpital rule we derive some exact slow roll conditions, valid for late time. It is then easy to obtain the scaling solution potentials and determine if the potential leads to an accelerating universe.

This work is organized as follows. In section III we present an overview of the tachyon field $T$. In section III we use the definition of the mass of a scalar field as the pole of the propagator to determine the mass of the tachyon. In section IV] we derive the transformation of the field $T$ in order to have a canonical normalized scalar field $\phi$ while in section $\nabla$ we study the model independent behavior of $\phi$ and we present some examples. Finally in section VI we use the L'Hôpital rule to study the late time behavior of the scalar field and we conclude in section VII

\section{TACHYON FIELD $T$}

The string tachyon field $T$ is given in terms of a BornInfeld "BI" Lagrangian [3],

$$
L=-V(T) \sqrt{1-\partial_{\mu} T \partial^{\mu} T},
$$

with $V(T)$ the potential. The potential is in principle arbitrary but if we want to describe a stringy tachyon then $V$ has a maximum at the origin $T=0$ with positive energy. The evolution of $T$ can be obtained by solving the equation of motion of eq.(1) and for an homogenous field in a Minkowski metric is given by

$$
\frac{\ddot{T}}{1-\dot{T}^{2}}+\frac{V_{T}}{V}=0
$$


where $\dot{T} \equiv d T / d t$ and $V_{T} \equiv d V / d T$. From eq.(2) the second term could define an effective potential

$$
F(T) \equiv \log [V(T)],
$$

with $d F / d T=V_{T} / V$. The potential $F$ has been widely used in the literature as the effective potential but as we will show neither $F$ nor $V$ are the correct effective potential for $T$ [2].

The energy density and pressure are given by

$$
\rho=\frac{V(T)}{\sqrt{1-\dot{T}^{2}}}, \quad p=L=-V(T) \sqrt{1-\dot{T}^{2}} .
$$

In terms of $\rho$ we can write the pressure as

$$
p=-\frac{V^{2}}{\rho}=w \rho
$$

with the effective equation of state given by $w=$ $-V^{2} / \rho^{2}$. From eq.(5) we see that the tachyon field can be treated as a Chaplygin gas for a flat potential $V$ or as quintessence with $w=-V^{2} / \rho^{2}$.

The equation of motion (2) implies that $\dot{\rho}=0$, i.e. the energy density is conserved, and $\dot{T}$ becomes a function of $T$, which from eq.(4) is

$$
\dot{T}^{2}=1-\frac{V(T)^{2}}{\rho^{2}} .
$$

If at the origin $T=T_{i}=0$ we set up the initial condition $\dot{T}_{i}=0$, then from eq. (44) we see that the energy density takes the constant value $\rho=V_{i} \equiv V\left(T_{i}\right)$ and $V_{i}$ represents the maximum value of the potential $V$, i.e. $V(T) \leq V_{i}$ for all $T$. Furthermore, if the minimum of the potential vanishes, $\left.V\right|_{\min }=0$, then at this point the kinetic energy becomes $\left.\dot{T}^{2}\right|_{\min }=1$. Therefore, if we want to have a canonically normalized field which includes the late time behavior of $T$, specially important for dark energy considerations, then the field transformation should necessarily be valid for large values of $\dot{T}$.

\section{MASS}

The mass of a particle enters in different phenomenological processes such as particle decay or reheating. Therefore, it is important to determine what is the mass of the tachyon field $T$. Naively, the mass of a scalar field is given by the second derivative of the potential $V(\phi)$ w.r.t. the field, e.g. $m^{2}=d V^{2} / d \phi^{2}$. However, this statement is only valid if the scalar field has a canonical kinetic term. Of course, this is not the case for the tachyon field $T$. Even if we consider the effective potential $F$, c.f. eq. (33), the mass of $T$ is neither given by $d^{2} V / d T^{2}$ nor by $d^{2} F / d T^{2}=V_{T T} / V-V_{T}^{2} / V^{2}$.

The mass of a particle is simply given by the pole of the propagator [5]. So in order to determine what the mass of the tachyon field is, we need to expand the Lagrangian
$L\left(\phi, \partial_{\mu} \phi\right)$ up to second order in the field perturbations $\phi(t, x) \simeq \phi_{o}(t)+\delta \phi(t, x)$. For quite arbitrary potentials and kinetic terms with $L\left(\phi, \partial_{\mu} \phi \partial^{\mu} \phi\right)$, the second order term can be put in the form

$$
\begin{aligned}
\int d x^{4} \delta L & =\int d x^{4}\left[A(t) \delta \dot{\phi}^{2}+B(t) \delta \phi \delta \dot{\phi}+\right. \\
& \left.+C(t) \delta \phi^{2}+D(t) \delta \phi^{2}\right]
\end{aligned}
$$

with $\delta \phi^{\prime}=\partial(\delta \phi) / \partial x$ and

$$
\begin{aligned}
& A=\frac{1}{2} \frac{\partial^{2} L}{\partial \dot{\phi}^{2}}, \quad B=\frac{\partial^{2} L}{\partial \dot{\phi} \partial \phi}, \\
& C=\frac{1}{2} \frac{\partial^{2} L}{\partial \phi^{\prime 2}}, \quad D=\frac{1}{2} \frac{\partial^{2} L}{\partial \phi^{2}},
\end{aligned}
$$

with all other second derivative terms being zero. After integrating by parts and dropping surface terms we get

$$
\int d x^{4} \delta L=-\int d x^{4} A \delta \phi\left(\partial_{t}^{2}+\frac{C}{A} \partial_{x}^{2}+m^{2}\right) \delta \phi,
$$

with the mass of the scalar field given by

$$
m^{2}=-\frac{1}{A}\left(D+\frac{\ddot{A}}{2}-\frac{\dot{B}}{2}\right) .
$$

Clearly from eq. (10), $m^{2}$ represents the pole of the propagator $\left(\partial_{t}^{2}+\frac{C}{A} \partial_{x}^{2}+m^{2}\right) \Delta_{F}(x)=-\delta^{4}(x)$.

In the case of a canonical normalized scalar field with Lagrangian $L=\left(\partial_{\mu} \varphi\right)^{2} / 2-W(\varphi)$ the coefficients of eq.(7) are

$$
\begin{gathered}
A=\frac{1}{2}, \quad B=0, \\
C=-\frac{1}{2}, \quad D=-\frac{1}{2} \frac{d^{2} W}{d \varphi^{2}} .
\end{gathered}
$$

The term $A=1 / 2$ defines a canonically normalized field and the mass is given by the usual expression $m^{2}=$ $-D / A=d^{2} W / d \varphi^{2}$.

In the case of the tachyon field $T$ given in eq.(11), one has

$$
\begin{gathered}
A=\frac{\rho^{3}}{2 V^{2}}, \quad B=\frac{\rho V_{T} \dot{T}}{V}, \\
C=-\frac{\rho}{2}, \quad D=-\frac{V V_{T T}}{2 \rho},
\end{gathered}
$$

which gives a mass term

$$
m^{2}(T)=\frac{V_{T T}}{V}-\frac{V_{T}^{2}}{V^{2}}\left(3-\frac{V^{2}}{\rho^{2}}\right),
$$

were we have used eq. (11). As mentioned above, the pole of the propagator, which gives the mass of the tachyon, does not coincide with $d^{2} V / d T^{2}$ nor with $d^{2} F / d T^{2}=$ $V_{T T} / V-V_{T}^{2} / V^{2}$.

The term proportional to the space dimensions $\partial_{x}^{2}$ is given by $C / A=-V^{2} / \rho^{2}$ giving a Carrollian type metric (for further reading see $[\underline{6}]$ ). The phase velocity of the perturbations is given by $v_{p h}^{2}=-C / A=V^{2} / \rho^{2}$ and it vanishes at $V=0$. 


\section{NORMALIZED SCALAR FIELD}

We are now interested in finding a field transformation which gives a canonical tachyon field valid for arbitrary values of $\dot{T}$. A scalar field with canonical kinetic terms in the limit of small kinetic term $(|\dot{T}| \ll 1)$ has been studied in the literature, giving a Lagrangian $L \simeq V(T)\left(1-\dot{T}^{2} / 2\right)$ with $A=V / 2$ as defined in eq.(8), and suggesting the transformation [2]

$$
\dot{\varphi}=\sqrt{V} \dot{T} .
$$

This transformation gives a Lagrangian $L \simeq \dot{\varphi}^{2} / 2-V$. However, clearly the field $\varphi$ is canonically normalized only in the limit of small kinetic terms and as discussed above the most interesting region is at late times where $V$ approaches zero and $\dot{T} \simeq 1$.

Instead, let us expand eq.(1) around an arbitrary value of $T_{o}(t)$ and $\dot{T}_{o}(t)$, i.e. $T(t)=T_{o}(t)+\delta T(t, x)$, and the second order term in $\delta \dot{T}$ is given by

$$
\delta L \simeq \frac{V\left(T_{o}\right)}{2\left(1-\dot{T}_{o}^{2}\right)^{3 / 2}} \delta \dot{T}^{2},
$$

which is just the term $A$ of eq. 113) since the energy density $\rho=V\left(T_{o}\right) / \sqrt{1-\dot{T}_{o}^{2}}$ is constant. From eq. 116) we suggest the new tachyon transformation

$$
\dot{\phi}=\sqrt{\frac{V\left(T_{o}\right)}{\left(1-\dot{T}_{o}^{2}\right)^{3 / 2}}} \dot{T}=\frac{\rho^{3 / 2}}{V} \dot{T}
$$

to obtain a canonical normalized tachyon field $\phi$. The transformation in eq. (17) reduces to that of eq. (15) when $|\dot{T}| \ll 1$ which implies that $\rho \simeq V$. However, in our case the transformation in eq. (17) is not only valid for small $\dot{T}$ but it is also valid for arbitrary values of $\dot{T}$.

Integrating eq.(17), using the fact that $\rho(t)$ is constant, we get

$$
\phi=\int \frac{\rho^{3 / 2} \dot{T}}{V} d t=\rho^{3 / 2} \int \frac{\dot{T}}{V} d t=\rho^{3 / 2} \int \frac{d T}{V} .
$$

The equation of motion for $\phi$ can be easily obtained from eq.(2) using eq.(17) (with $\rho$ constant) giving

$$
\begin{aligned}
\ddot{\phi}+\frac{\rho^{3}}{V^{3}} \frac{d V}{d \phi} & =0, \\
\ddot{\phi}+U_{\phi} & =0,
\end{aligned}
$$

with the effective potential $U(\phi)$ defined by $U_{\phi} \equiv$ $d U / d \phi=\left(\rho^{3} / V^{3}\right) d V / d \phi$. For $\rho$ constant we can integrate $U$ and we get an effective potential

$$
U=U_{o}-\frac{\rho^{3}}{2 V^{2}}
$$

with $U_{o}=3 \rho / 2$. The energy density in eq. (4) can be expressed in terms of $\phi$ and the effective potential $U$ as,

$$
\rho=\frac{V}{\sqrt{1-\dot{T}^{2}}}=\frac{V}{\sqrt{1-\left(V^{2} / \rho^{3}\right) \dot{\phi}^{2}}}=\frac{1}{2} \dot{\phi}^{2}+U(\phi) \text {. }
$$

The last equality in eq. (22) can be easily derived by extracting $\dot{\phi}^{2}$ from $\rho^{2}=V^{2} /\left(1-V^{2} \dot{\phi}^{2} / \rho^{3}\right)$ and comparing it with $\dot{\phi}^{2}=2(\rho-U)$. It is clear from the last equality in eq. (22) that the energy density $\rho$ in terms of $\phi$ and $U$ is that of a canonically normalized scalar field which can be derived from a Lagrangian

$$
L=\frac{1}{2} \dot{\phi}^{2}-U(\phi),
$$

and with an equation of motion given by eq. (19). The mass of the $\phi$ is given by

$$
M^{2}(\phi)=\frac{d^{2} U}{d \phi^{2}}=\frac{\rho^{3}}{V^{2}}\left(\frac{V_{\phi \phi}}{V}-3 \frac{V_{\phi}^{2}}{V^{2}}\right),
$$

where $V_{\phi} \equiv d V / d \phi$. In terms of the field $T$ using eq. (17) we can express $U_{\phi}$ and $U_{\phi \phi}$ as

$$
\begin{aligned}
U_{\phi} & =\frac{\rho^{3}}{V^{3}} \frac{d V}{d \phi}=\frac{\rho^{3}}{V^{3}} \frac{d T}{d \phi} \frac{d V}{d T}=\frac{\rho^{3 / 2}}{V^{2}} \frac{d V}{d T}, \\
M^{2} & =\frac{d^{2} U}{d \phi^{2}}=\frac{\rho^{3}}{V^{2}}\left(\frac{V_{\phi \phi}}{V}-3 \frac{V_{\phi}^{2}}{V^{2}}\right)=\left(\frac{V_{T T}}{V}-2 \frac{V_{T}^{2}}{V^{2}}(£ 6)\right.
\end{aligned}
$$

If we substitute eq.(17) into eq.(1) we get a Lagrangian $L=-V(\phi) \sqrt{1-V(\phi)^{2} \dot{\phi}^{2} / \rho^{3}}$, with $\rho$ constant, and if we expand to second order in perturbations of $\phi$ around an arbitrary value $\phi_{o}(t)$, the coefficients $A, B, C, D$, as defined in eq.(7), are now given by

$$
\begin{gathered}
A=\frac{1}{2}, \quad B=\frac{\dot{\phi} V_{\phi}}{V}\left(3-\frac{2 V^{2} \dot{\phi}^{2}}{\rho^{3}}\right), \\
C=-\frac{V^{2}}{2 \rho^{2}}, \quad D=-\frac{V_{\phi \phi} \rho}{2 V}\left(1-2 V^{2} \dot{\phi}^{2}\right)+\frac{V_{\phi}^{2} \dot{\phi}^{2}}{2 V^{2}}\left(3-2 V^{2} \dot{\phi}^{2}\right) .
\end{gathered}
$$

In this case we have $A=1 / 2$ and $C / A=-V^{2} / \rho^{2}$ showing that the field $\phi$ has canonical normalized kinetic term (in a Carrollian metric). The mass of $\phi$ given as the pole of the propagator using eqs.(11) and (27) gives a mass which is equal to that of eq. (24).

We have shown that to first and second order the homogenous part of the Lagrangian $L=$ $-V(\phi) \sqrt{1-V^{2} \dot{\phi}^{2} / \rho^{3}}$ and $L=\frac{1}{2} \dot{\phi}^{2}-U(\phi)$ are equivalent.

\section{GENERAL ANALYSIS}

Let us now study some general properties of the tachyon field $\phi(T)$. Without loss of generality we take the origin at $T=0$ and we assume $V_{i}=V(T=0) \neq 0$. If the field $T$ is a tachyon at the origin then $V_{T}(T=0)=0$ and $V_{T T}(T=0)>0$. We can see that the tachyonic property of $T$ is transmitted to $\phi$ and its potential $U(\phi)$, since at the origin $\phi=0$ and from eqs.(21), (25) and (26) we have 
$U(\phi=0)=\rho=V_{i}$ and $U_{\phi}(0)=V_{T} /\left.V_{i}^{1 / 2}\right|_{\text {min }}=0$ and $U_{\phi \phi}(0)=V_{T T} /\left.V_{i}\right|_{\min }>0$.

The potential $U=U_{o}-\rho^{3} / 2 V^{2}$ diverges and is unbounded from below at $V \rightarrow 0$. In terms of the potential $U$ and using eq.(17) the value of $\dot{\phi}$ is

$$
\dot{\phi}^{2}=2(\rho-U)=\rho\left(\frac{\rho^{2}}{V^{2}}-1\right) .
$$

At the origin one has $V\left(T_{i}\right)=V_{i}=\rho$ and $\dot{\phi}=0$ while at the minimum $V(T)=0$ one has $|\dot{\phi}|=\infty$. Even though the potential is unbounded from below the total energy density $\rho$ remains constant. The conclusion is that at the minimum the quantities $U(\phi)$ and the field $\phi, \dot{\phi}$ tend to an infinite value, showing that $\phi$ has a runaway behavior and does not oscillate at the minimum $V=0$. However, there is no stable configuration. This could be interpreted as the decay of the unstable brane-antibrane system represented by the tachyonic potential.

\section{A. Examples}

In this section we will analyze a few interesting examples. First we will study the potentials $V=$ $V_{i} \operatorname{Exp}\left[-T^{2} / 2\right]$ [3] and $V=V_{i} / \cosh [T]$ [8]. Both of them have been conjectured to represent the tachyon field for a Dp-brane system in superstrings. Later we will study a cubic potential which is unbounded from below for negative $T$ and has been related to Dp-brane system in bosonic strings.

\section{Potential $V=V_{i} \operatorname{Exp}\left[-T^{2} / 2\right]$}

The potential $V=V_{i} \operatorname{Exp}\left[-T^{2} / 2\right]$ has been widely studied in the literature [1], 3]. It has a maximum at $T=0$ while it goes to zero at large $T$ with vanishing potential.

The normalized tachyon field $\phi$ in terms of $T$, as given by eq. (18), is

$$
\phi=\sqrt{\frac{\pi}{2}} E_{r f i}\left[\frac{T}{\sqrt{2}}\right],
$$

and can be seen in figure (11). Notice that at $T \rightarrow \pm \infty$ one has the limit $\phi \rightarrow \pm \infty$. The effective potential $U(\phi)$ is unbounded from below for $V(T) \rightarrow 0$ and we show in fig.(2) the behavior of $U, V$ and $F=\log [V]+c$, with $c$ a constant such that $F\left(T_{i}\right)=V\left(T_{i}\right)$ given by a solid, dashed and dotted lines respectively. The effective potentials $F$ is also unbounded from below for $V(T) \rightarrow 0$.

The mass of the normalized tachyon field $\phi$ given by eq.(26), the mass of $T$ given by eq.(14), and the second derivative of $V$ and $F$ are then

$$
\begin{aligned}
M^{2} & =-\left(T^{2}+1\right), m^{2}=T^{2}\left(e^{-T^{2}}-2\right)-1, \\
V_{T T} & =e^{-T^{2} / 2}\left(T^{2}-1\right), \quad F_{T T}=-1
\end{aligned}
$$

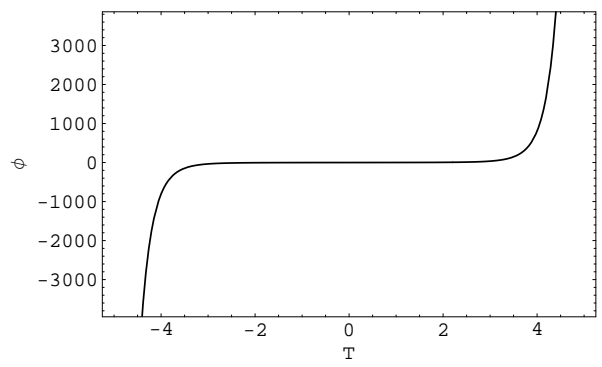

FIG. 1: We show the normalized tachyon field $\phi$ as a function of $T$ for $V=V_{i} \operatorname{Exp}\left[-T^{2} / 2\right]$.

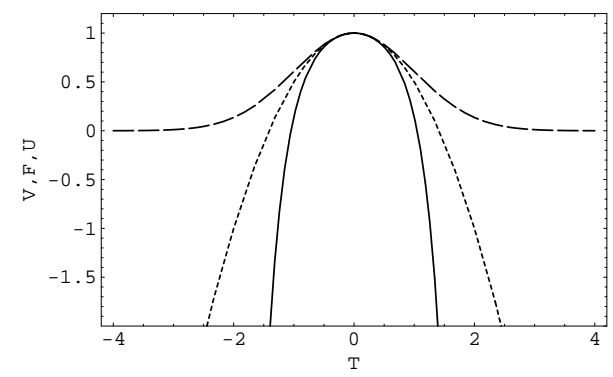

FIG. 2: We show the potentials $U, V, F$ as a function of $T$ (solid, dashed and dotted lines respectively) for $V=$ $V_{i} \operatorname{Exp}\left[-T^{2} / 2\right]$.

and are shown in fig.(3) (solid, dashed, dash-dotted and dotted, respectively). The mass $M^{2}, m^{2}, F_{T T}$ are negative for all values of $T$ and $M^{2}, m^{2}$ diverge at $T \rightarrow \pm \infty$ or equivalently at $V(T) \rightarrow 0$. The infinite mass implies that the field is no longer a dynamical field at these points. On the other hand, the quantity $V_{T T}$ is negative at the origin and tends to zero from above at $T= \pm \infty$.

\section{Potential $V=V_{i} / \cosh [T]$}

Let us now study the potential $V=V_{i} / \operatorname{Cosh}[T]$ [8], which has been also widely analyzed in the literature. This potential has a maximum at the origin $T=0$, is never negative, and goes to zero at large $T$.

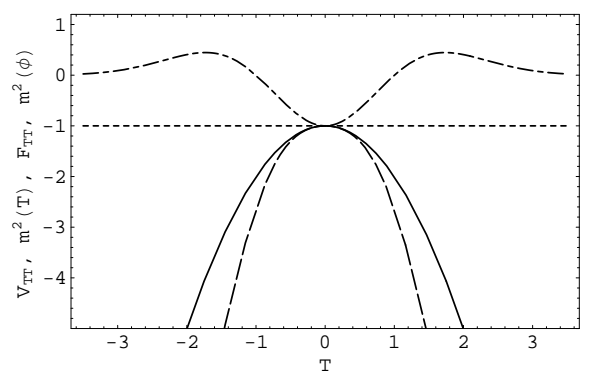

FIG. 3: We show the mass $M^{2}, m^{2}$ and the second derivatives $V_{T T}, F_{T T}$ as a function of $T$ (solid, dashed, dash-dotted and dotted, respectively) for $V=V_{i} \operatorname{Exp}\left[-T^{2} / 2\right]$. 


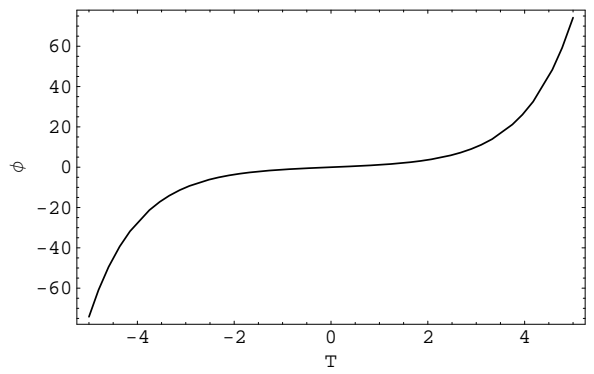

FIG. 4: We show the normalized tachyon field $\phi$ as a function of $T$ for a potential $V=V_{i} / \cosh [T]$.

Using eq. (18) the normalized tachyon field $\phi$ in terms of $T$ is

$$
\phi=\sinh [T],
$$

and can be seen in figure (4). For $T \rightarrow \pm \infty$ one has the limit $\phi \rightarrow \pm \infty$, as expected. The effective potentials $U(\phi)$ and $F(T)$ are unbounded from below for $V(T) \rightarrow 0$ while $V(T) \geq 0$ for all $T$. In fig.(15) we show the behavior of $U, V$ and $F$ given by the solid, dashed and dotted lines respectively. The behavior of the normalized scalar field $\phi$ and the potentials $U, F$ are very similar in the two examples considered, namely $V=V_{i} \operatorname{Exp}\left[-T^{2} / 2\right]$ and $V=V_{i} / \cosh [T]$.

The mass of the normalized tachyon field $\phi$ given by eq.(26), the mass of $T$ given by eq.(14), and the second derivative of $V$ and $F$ are in this case

$$
\begin{aligned}
M^{2} & =-1, \quad m^{2}=-\frac{1}{4} \frac{(\cosh [4 T]+3)}{\cosh [T]^{4}}, \\
V_{T T} & =\frac{1}{2} \frac{(\cosh [2 T]-3)}{\cosh [T]^{3}}, \quad F_{T T}=-\frac{1}{\cosh [T]^{2}},
\end{aligned}
$$

and are shown in fig. (6) (solid, dashed, dash-dotted and dotted, respectively). The mass $M^{2}$ is negative and constant, while $m^{2}, F_{T T}$ are negative for all values of $T$ and approach zero from below for $T \rightarrow \infty$. The quantity $V_{T T}$ is negative at the origin and tends to zero from above at $T= \pm \infty$.

Contrary to the case $V=V_{i} \operatorname{Exp}\left[-T^{2} / 2\right]$ for the potential $V=V_{i} / \cosh [T]$ the mass of $\phi$ and $T$, i.e. $M^{2}$ and $m^{2}$, do not diverge even though the effective potentials $U, F$ diverge at large $T$.

$$
\text { 3. Potential } V=T^{3}+T^{2}-4 / 27
$$

We will now analyze the potential $V=T^{3}+T^{2}-$ $4 / 27$. This kind of potential has been suggested [3] to parameterize a tachyon field of a unstable bosonic Dpbrane systems. The potential has a maximum at the origin $T=0$, a minimum at $T=2 / 3$, is unbounded from below for negative $T$ and $V(T)$ vanishes at $T=2 / 3,-1 / 3$ as seen from fig.(7).

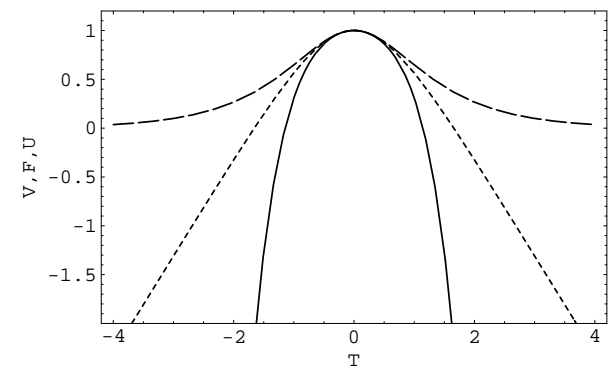

FIG. 5: We show the potentials $U, V, F$ as a function of $T$ (solid, dashed and dotted lines respectively) for $V=$ $V_{i} / \cosh [T]$.

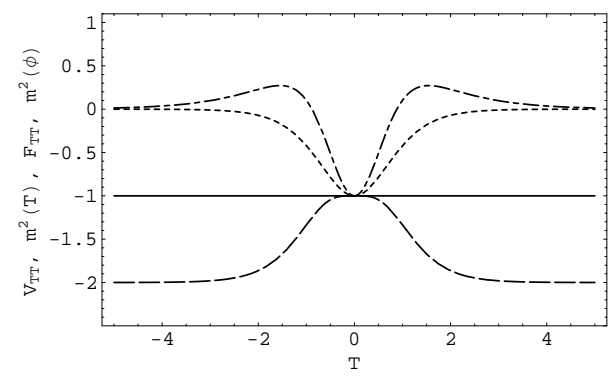

FIG. 6: We show the mass $M^{2}, m^{2}$ and the second derivatives $V_{T T}, F_{T T}$ as a function of $T$ (solid, dashed, dash-dotted and dotted, respectively) for $V=V_{i} / \cosh [T]$.

The normalized tachyon field $\phi$ in terms of $T$, as given by eq. (18), is

$$
\phi=\frac{8}{81 \sqrt{3}}\left(\frac{9 T}{2(2-3 T)}+\log \left[\frac{2(1+3 T)}{2-3 T}\right]\right),
$$

and can be seen in figure (8). It is clear that $\phi$ has a pole at $T=2 / 3$ and $T=-1 / 3$ where $|\phi| \rightarrow \infty$. So, even though the potential $V(T)$ is defined for all values of $T$, the normalized tachyon $\phi$ is only defined in the interval $-1 / 3 \leq T \leq 2 / 3$ which implies that the field $\phi$ does not know of the unboundedness of $V(T)$ for negative $T$. However, once again the effective potential $U(\phi)$ and $F$ are unbounded from below for $V(T) \rightarrow 0$ and we show in fig.(9) the behavior of $U, V$ and $F$ given by a solid, dashed and dotted lines, respectively.

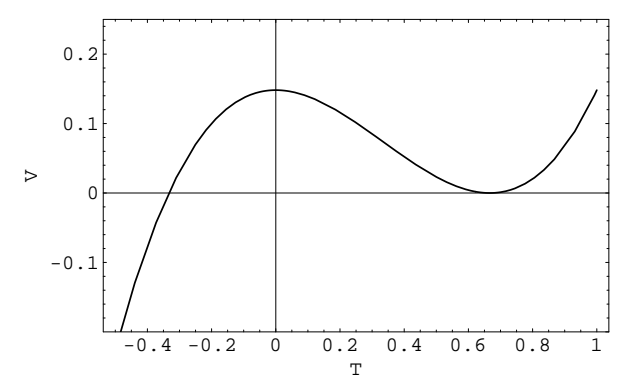

FIG. 7: We show the potential $V=T^{3}+T^{2}-4 / 27$ as a function of $T$. 


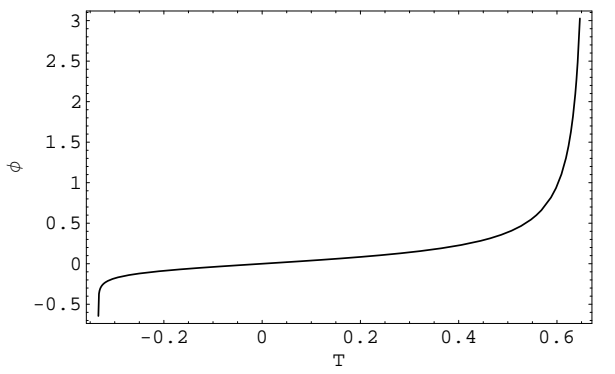

FIG. 8: We show the normalized tachyon field $\phi$ as a function of $T$.

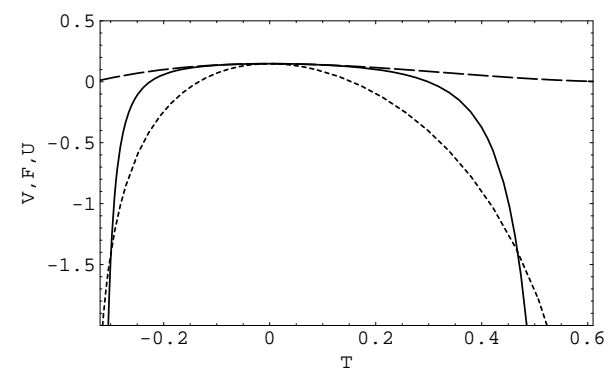

FIG. 9: We show the potentials $U, V, F$ as a function of $T$ (solid, dashed and dotted lines respectively).

The mass of the normalized tachyon field $\phi$ given by eq.(26), the mass of $T$ given by eq.(14), and the second derivative of $V$ and $F$ are then

$$
\begin{aligned}
M^{2} & =-\frac{54\left(1+18 T^{2}\right)}{(2-3 T)^{2}(1+3 T)^{2}} \\
m^{2} & =\frac{27\left[-32-576 T^{2}+5832 T^{4}(T-1)\right]}{16(2-3 T)^{2}(1+3 T)^{2}} \\
& +\frac{27 \times 19683 T^{6}(1-T)^{2}}{16(2-3 T)^{2}(1+3 T)^{2}} \\
V_{T T} & =6 T-2 \\
F_{T T} & =-\frac{27\left(2+9 T^{2}\right)}{(2-3 T)^{2}(1+3 T)^{2}}
\end{aligned}
$$

and are shown in fig.(10) (solid, dashed, dash-dotted and dotted, respectively). The mass $M^{2}, m^{2}$ and $F_{T T}$ are negative for all values of $T$ and have a pole at $T=2 / 3,-1 / 3$ or equivalently $V(T) \rightarrow 0$. The infinite mass implies that the field is no longer dynamical at these points. The quantity $V_{T T}$ is only positive for $T>1 / 3$.

\section{LATE TIME BEHAVIOR}

We will analyze the late time behavior of the normalized tachyon field in a BI Lagrangian. We are also interested in determining if we can obtain an accelerating universe at late times. In order to do this, we will use the L'Hôpital rule to derive the slow roll conditions which will given by exact relationships. Tachyonic slow roll conditions has been studied in [2].

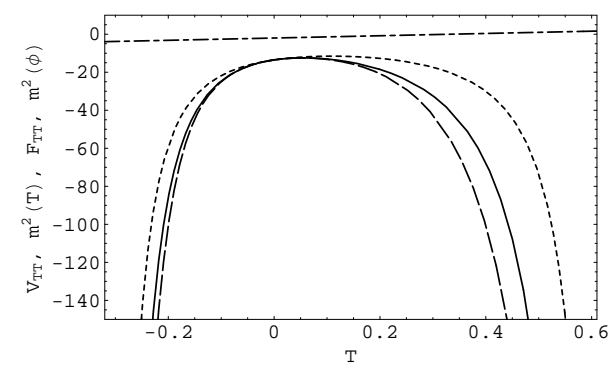

FIG. 10: We show the mass $M^{2}(\phi), m^{2}(T)$ and the second derivatives $V_{T T}, F_{T T}$ as a function of $T$ (solid, dashed, dashdotted and dotted, respectively).

\section{A. Slow roll conditions and L'Hôpital rule}

\section{Canonical scalar field $\varphi$}

As a matter of presentation purpose let us first begin with a canonically normalized field and then we will study the case of a BI type lagrangian. In this first case the energy density is given by $\rho=E_{k}+V$ and the pressure by $p=E_{k}-V$ with $E_{k}=\dot{\varphi}^{2} / 2$ the kinetic energy and $V(\varphi)$ the scalar potential. A general analysis can be found in 9]. The evolution of $\rho$ and acceleration of the scale factor $a(t)$ of the universe are given in terms of $\rho, p$ by

$$
\begin{aligned}
\dot{\rho} & =-3 H(\rho+p)=-6 H \rho(1-X), \\
\frac{\ddot{a}}{a} & =-\frac{1}{6}(\rho+3 p)=-\rho\left(\frac{2}{3}-X\right),
\end{aligned}
$$

with $H \equiv \dot{a} / a$. We have defined the quantity $X \equiv V / \rho$ and we expressed $E_{k}=\rho-V=\rho(1-X)$ in the last part of eqs. (41) and (42). The quantity $X$ is constrained by the values $1 \geq X \geq 0$. From eq.(42) we see that if $X$ is larger than $2 / 3$ then we have an accelerating universe. The equation of state parameter $w$ is given by $w=p / \rho=$ $1-2 X$ and if $X$ is constant then we have a scaling solution with $\rho=\rho_{o}\left(a / a_{o}\right)^{-6(1-X)}$.

We will consider only potentials that vanish at the minimum $V_{\min }=0$ (if $V_{\min }>0$ then it is easy to show that the dynamics always leads to inflation and for $V_{\min }<0$ we have a non-inflationary universe and in fact a big crunch [10]). For $V=0$ with $\rho \neq 0$, i.e. $X=0$, we see from eq. (42) that $\varphi$ does not lead to an inflationary universe. However, from eq. (41) we have in this case a negative $\dot{\rho}$ and therefore $\rho$ decreases until it reaches $\rho=0$. This arguments shows that the late time behavior is the expected one $V \rightarrow 0, \rho \rightarrow 0$ but the limit $X=V / \rho$ is model dependent. Since the limits of $V$ and $\rho$ are zero we can use the L'Hôpital rule to determine the asymptotic (late time) value,

$$
\lim \left(X=\frac{V}{\rho}\right)=\lim \left(\frac{\dot{V}}{\dot{\rho}}\right),
$$


and since the limit $\dot{\rho}=0$, if $\dot{V}$ also vanishes then

$$
\lim X=\lim \left(\frac{\ddot{V}}{\ddot{\rho}}\right) .
$$

Now, taking $H^{2}=\rho / 3$, the equation of motion $\ddot{\varphi}=$ $-V^{\prime}-3 H \dot{\varphi}$ and using eq. (41) the equations (43) and (44) give the constraints

$$
\begin{aligned}
\frac{V^{\prime}}{V} & =\sqrt{6(1-X)}<\sqrt{2}, \\
\frac{V^{\prime \prime}}{V} & =6(1-X)<2,
\end{aligned}
$$

where we have used eq. (45) to write the second derivative of the potential as $\ddot{V}(\varphi)=V^{\prime \prime} \dot{\varphi}^{2}+V^{\prime} \ddot{\varphi}=2(1-X) \rho\left(V^{\prime \prime}+\right.$ $3 V(1-X))$ to derive eq.(46).

Equations (45) and (46) are the slow roll conditions at late time (i.e. for vanishing $V, \rho$ ) and are exact equations in this limit. An inflationary universe must satisfied the condition $X>2 / 3$ which gives the last inequalities in eqs.45). We have determined that exact value of $V^{\prime} / V, V^{\prime \prime} / V$ at which the universe no longer inflates at late time. It is easy to see that the limit is satisfied for a potential of the form $V=V_{o} \exp [6(1-X) \phi]$ and the exponent must be smaller than two for $V$ to inflate. This potential gives the scaling solution (i.e. $X=$ cte) and $\rho \propto\left(a / a_{o}\right)^{-6(1-X)}$.

A late time inflation, i.e. dark energy, requires $X>$ $2 / 3$ and in the limit $X \rightarrow 1$ from eqs.(45) one has $V^{\prime} / V \rightarrow 0, V^{\prime \prime} / V \rightarrow 0$. For a potential $V=\varphi^{\alpha}$ a period of late time inflation is simply obtained for a negative $\alpha$.

\section{Tachyon field $T$}

Now, let us repeat the analysis for the tachyon field with Lagrangian (1) in a FRW metric. The equation of motion for $T$ is $\ddot{T} /\left(1-\dot{T}^{2}\right)+3 H \dot{T}+V_{T} / V=0$ and the energy density and pressure are given by eqs.(4). The evolution of $\rho$ and the acceleration of the universe are now given by

$$
\begin{aligned}
\dot{\rho} & =-3 H(\rho+p)=-3 H \rho\left(1-X^{2}\right), \\
\frac{\ddot{a}}{a}=-\frac{1}{6}(\rho+3 p) & =-\frac{1}{2} \rho\left(\frac{1}{3}-X^{2}\right),
\end{aligned}
$$

with $1 \geq X=V / \rho \geq 0$. In this case an inflationary universe requires $X^{2}>1 / 3$. It is clear that the r.h.s. of eq. (47) is non positive which implies that $\rho$ decreases or it remains constant in time. The constant value of $\rho$ takes place either for $\rho=0$ or at $X^{2}=V^{2} / \rho^{2}=1$. As in the canonical case, if $V(T)=0$ with $\rho \neq 0$ then $X=0$ and from eq.48) it is easy to see that the tachyon field does not inflate the universe. However, in the limit $V \rightarrow 0$ with $X \neq 1$ the energy density decreases and $\rho \rightarrow 0$ at late times.

Since $V$ and $\rho$ tend to zero then we can use the L'Hôpital rule to determine the asymptotic behavior of
$X$ and using the equation of motion of $T$ the eqs. (43) and (44) give the constraints

$$
\begin{aligned}
\mid \frac{V_{T}}{V^{3 / 2}} & =-\sqrt{3\left(\frac{1}{X}-X\right)} \mid<\sqrt{2 \sqrt{3}}, \\
\frac{V_{T T}}{V^{2}} & =\frac{9}{2}\left(\frac{1}{X}-X\right)<3 \sqrt{3} .
\end{aligned}
$$

Eqs. (49) and (50) give the exact late time limit for tachyonic potentials and the inequality must hold for the universe to inflate (i.e. $X^{2}>1 / 3$ ). A scaling solution requires $X=$ cte and from eqs. (49) we obtain the potential $V=V_{o} / T^{2}$. This potential has been widely studied [12] and here we have derived it through a very simple and powerful tool, the L'Hôpital rule. Our analysis reproduces the result for a scaling solution given in 11], namely $\lambda \equiv-V_{T} / V^{3 / 2}$ constant and $\Gamma \equiv V V_{T T} / V_{T}^{2}=3 / 2$. Using the potential $V=V_{o} / T^{2}$ in eqs. (49) the constant $V_{o}$ is given in terms of $X$ by $V_{o}=4 X / 3\left(1-X^{2}\right)$ and an inflationary universe requires $V_{o} \geq 2 / \sqrt{3}$ for $X^{2} \geq 1 / 3$. Contrary to a canonically normalized fields were an inverse power potential always leads to inflation at late times in this case the constant term determines whether the universe inflates or not.

If $X=0$ then the r.h.s. of eqs. (49) and (50) is infinite and taking the ansatz $V=T^{\alpha}$, giving $V_{T} / V^{3 / 2}=$ $T^{-(2+\alpha) / 2}$ and $V_{T T} / V^{2}=T^{-(2+\alpha)}$, eqs. (49) and (50) are satisfied for $\alpha>0$ with $V(T \rightarrow 0) \rightarrow 0$ or for $\alpha<-2$ in the limit $V(T \rightarrow \infty) \rightarrow 0$. On the other hand, if $X=1$ then the r.h.s. of eqs. (49) and (50) vanish which implies $0 \geq \alpha>-2$ for $V(T \rightarrow \infty) \rightarrow 0$. We have, therefore, shown that for inverse power potentials only the models with $0 \geq \alpha \geq-2$ lead to a late time inflation, i.e. dark energy.

\section{B. Asymptotic Limit for $\phi$}

Now, we would like to study how the normalized tachyon field $\phi$ defined by eq.(17) behaves at late time. We will assume that the origin is at $T=0$ and the potential $V(T)$ vanishes at $T=T_{m}$, i.e. $V\left(T_{m}\right)=0$, where $T_{m}$ can take either an infinite or a finite value. The potential may have a minimum at $T_{m}$ but it is not relevant if $V\left(T_{m}\right)$ is a minimum or not.

Let us first begin the study in a Minkowski metric. In this case we have a constant $\rho$ and from eq.(17) it is easy to see that in the limit $V\left(T \rightarrow T_{m}\right) \rightarrow 0$ one has

$$
\phi=\int \rho^{3 / 2} \frac{d T}{V(T)}=\rho^{3 / 2} \int \frac{d T}{V(T)} \rightarrow \infty,
$$

since the integrand diverges. This implies that the normalized scalar field $\phi$ is constraint between the zeros of the potential $V(T)$ and never reaches the region of $T>T_{m}>0$ (or $T<T_{m}$ for negative $T_{m}$ ) and $\phi$ always reaches an infinite value at $V(T)=0$, i.e. the field $\phi$ is only defined in the interval $T_{m 1} \leq T \leq T_{m 2}$, with 
$V\left(T_{m 1}\right)=V\left(T_{m 2}\right)=0$, and $\phi$ takes the whole range of values $-\infty \leq \phi \leq \infty$. This result contrast with the one obtained by using the transformation in eq. (15) where finite values for $\varphi$ are obtained in the limit $V \rightarrow 0$. However, we would like to point out again that the transformation (15]) gives a canonical scalar field only for small values of $\dot{T}$ and not for values of $\dot{T} \simeq 1$ where $V$ approaches zero.

The constraint on the interval on the tachyon field $T_{m 1} \leq T \leq T_{m 2}$, with $V\left(T_{m 1}\right)=V\left(T_{m 2}\right)=0$ is quite important for models with finite values $T_{m}$ as in a cubic potential $V=T^{3}+T^{2}-c,(c>0)$, where it has a local minimum for $T>0$ and is unbounded from below for $T<0$. This kind of potentials, obtained from Dpbrane in bosonic string theory, have not been thoroughly analyzed because for $T<0$ the potential $V(T)$ it is argued that the potential is unbounded from below and for the minimum at positive $T$ the system is unstable (a large growth of perturbations when $T$ oscillates around $\left.T_{m}>0\right)$. However, the physical field $\phi$ does not reach the regions outside the zeros of the potential $V(T)$ and therefore does not oscillate around the minimum with $T_{m}>0$ nor does it feel the unboundedness of the potential for negative $T$. The fact that $\phi$ does not oscillate around the minimum is what one would expect from a string motivated tachyon [3].

\section{Asymptotic Limit for $\phi$ in FRW}

In the case of a FRW metric, the energy density is no longer constant and we cannot take $\rho$ out of the integral in eq.(18). However, we can still easily investigate the asymptotic value of $\phi$ for $V(T) \rightarrow 0$ using the L'Hôpital rule, as in the previous section. The relevant quantity is the integrand $Y \equiv \rho^{3 / 2} / V(T)$ of eq.(18). Similar to eqs.(43) and (44) we have in this case

$$
\lim \left(Y=\frac{\rho^{3 / 2}}{V}\right)=\lim \left(\frac{(3 / 2) \dot{\rho} \rho^{1 / 2}}{\dot{V}}\right),
$$

with $\dot{V}=V_{T} \dot{T}$. Using eqs. (47) and (49), equation (52) becomes

$$
\lim Y=\lim \frac{3}{2} Y,
$$

which is only satisfied if $Y=\rho^{3 / 2} / V=0$ or $Y=\infty$. Since $Y=\sqrt{\rho} / X=\sqrt{V} / X^{3 / 2}$ and $0 \geq X=V / \rho \geq 1$ a value of $X \neq 0$ gives $Y \rightarrow 0$. This is the case for any model that leads to late time inflation since $X$ must be larger than $2 / 3$. In this case, the integrand in eq.(18) vanishes at late times and a finite value of $\phi$ is obtained as long as $\int Y d T \simeq\left(\int \sqrt{V} d T\right) / X^{3 / 2}$ is finite in the limit of vanishing $V$ (we have taken $X$ out of the integral since in the late time limit it approaches a constant value). This will happen for models with a vanishing potential $V(T)$ at a finite value of $T$. On the other hand, if $X=0$, which represents a model with no late time inflation, the limiting value of $Y$ is model dependent.

\section{SUMMARY AND CONCLUSIONS}

We have analyzed the tachyon field motivated by brane-antibrane interaction with an effective BI type lagrangian with potential $V(T)$. We have deduced the mass of the tachyon field $T$ as the pole of the propagator which is not given by the second derivative of $V$ nor by the second derivative of the effective potential $F \propto \log [V]$. The mass of $T$ is given by eq. (14).

Since $T$ does not have canonical kinetic terms we have generalized the commonly used transformation $\varphi=$ $\int \sqrt{V} d T$, which is only valid for small $\dot{T}$, to the transformation $\phi=\int\left(\rho^{3 / 2} / \sqrt{V}\right) d T$ valid for all $\dot{T}$ and which reduced to the transformation of $\varphi$ in the limit of small $\dot{T}$. We have derived the equation of motion for $\phi$ and determined the effective potential $U(\phi)$ given by eq.(21) and its mass eq.(24). We have shown that the normalized tachyon field $\phi$, in a Minkowski metric, is defined only in the region between the zeros of the original $V(T)$ potential and the field $\phi$ takes the whole range between $-\infty$ and $\infty$.

We have used the simple but powerful tool of L'Hôpital rule to derive exact slow roll conditions for late time inflation, i.e. dark energy. As a side result we derive the potential which leads to a scaling behavior for a standard scalar field and for a tachyon field.

\section{Acknowledgments}

A.M. would like to thank the kind hospitality of the DAMTP of the University of Cambridge and specially F. Quevedo during his short visit and the Royal-Society and the Academia Mexicana de Ciencias for financial support. A.M. would like to thank F. Quevedo for many useful discussions and early collaboration of this manuscript. A.M. would also like to thank the This work was also supported in part by CONACYT project $45178-\mathrm{F}$ and DGAPA, UNAM project IN114903-3.
[1] A. Sen, "Field theory of tachyon matter," Mod. Phys. Lett. A 17, 1797 (2002) arXiv:hep-th/0204143; A. Sen, "Remarks on tachyon driven cosmology," Phys. Scripta T117, 70 (2005) arXiv:hep-th/0312153; A. Sen, "Supersymmetric world-volume action for non-BPS D-branes," JHEP 9910, 008 (1999) arXiv:hep-th/9909062; M. R. Garousi, "Tachyon couplings on non-BPS D-branes and Dirac-BornInfeld action," Nucl. Phys. B 584, 284 (2000) arXiv:hep-th/0003122; M. R. Garousi, "On-shell 
S-matrix and tachyonic effective actions," Nucl. Phys. B 647, 117 (2002) arXiv:hep-th/0209068; M. R. Garousi, "Slowly varying tachyon and tachyon potential," JHEP 0305, 058 (2003) arXiv:hep-th/0304145; E. A. Bergshoeff, M. de Roo, T. C. de Wit, E. Eyras and S. Panda, "T-duality and actions for non-BPS D-branes," JHEP 0005, 009 (2000) arXiv:hep-th/0003221; J. Kluson, "Proposal for non-BPS D-brane action," Phys. Rev. D 62, 126003 (2000) arXiv:hep-th/0004106; D. Kutasov and V. Niarchos, "Tachyon effective actions in open string theory," Nucl. Phys. B 666, 56 (2003) arXiv:hep-th/0304045.

[2] M. Fairbairn and M. H. G. Tytgat, "Inflation from a tachyon fluid?," Phys. Lett. B 546, 1 (2002) arXiv:hep-th/0204070; A. Feinstein, "Power-law inflation from the rolling tachyon," Phys. Rev. D 66, 063511 (2002) arXiv:hep-th/0204140; S. Mukohyama, "Brane cosmology driven by the rolling tachyon," Phys. Rev. D 66, 024009 (2002) arXiv:hep-th/0204084; D. Choudhury, D. Ghoshal, D. P. Jatkar and S. Panda, "On the cosmological relevance of the tachyon," Phys. Lett. B 544, 231 (2002) arXiv:hep-th/0204204; G. Shiu and I. Wasserman, "Cosmological constraints on tachyon matter," Phys. Lett. B 541, 6 (2002) arXiv:hep-th/0205003; L. Kofman and A. Linde, "Problems with tachyon inflation," JHEP 0207, 004 (2002) arXiv:hep-th/0205121; M. Sami, "Implementing power law inflation with rolling tachyon on the brane," Mod. Phys. Lett. A 18, 691 (2003) arXiv:hep-th/0205146; G. N. Felder, L. Kofman and A. Starobinsky, "Caustics in tachyon matter and other Born-Infeld scalars," JHEP 0209, 026 (2002) arXiv:hep-th/0208019; S. Mukohyama, "Inhomogeneous tachyon decay, light-cone structure and D-brane network problem in tachyon cosmology," Phys. Rev. D 66, 123512 (2002) arXiv:hep-th/0208094; M. C. Bento, O. Bertolami and A. A. Sen, "Tachyonic inflation in the braneworld scenario," Phys. Rev. D 67, 063511 (2003) arXiv:hep-th/0208124; J. g. Hao and X. z. Li,
"Reconstructing the equation of state of tachyon," Phys. Rev. D 66, 087301 (2002) arXiv:hep-th/0209041; C. j. Kim, H. B. Kim and Y. b. Kim, "Rolling tachyons in string cosmology," Phys. Lett. B 552, 111 (2003) arXiv:hep-th/0210101; B. C. Paul and M. Sami, "A note on inflation with tachyon rolling on the Gauss-Bonnet brane," Phys. Rev. D 70, 027301 (2004) arXiv:hep-th/0312081 ; S. K. Srivastava, "Tachyon as a dark energy source," arXiv:gr-qc/0409074

[3] A. Sen, "Rolling tachyon," JHEP 0204, 048 (2002) arXiv:hep-th/0203211; A. Sen, "Tachyon matter," JHEP 0207, 065 (2002) arXiv:hep-th/0203265.

[4] A. V. Frolov, L. Kofman and A. A. Starobinsky, "Prospects and problems of tachyon matter cosmology," Phys. Lett. B 545, 8 (2002) arXiv:hep-th/0204187.

[5] S. Weinberg, "The Quantum Theory of Fields", Cmabridge Univ.Press (2000)

[6] G. W. Gibbons, "Thoughts on tachyon cosmology," Class. Quant. Grav. 20, S321 (2003) arXiv:hep-th/0301117;

[7] G. W. Gibbons, "Cosmological evolution of the rolling tachyon," Phys. Lett. B 537, 1 (2002) arXiv:hep-th/0204008.

[8] N. Lambert, H. Liu and J. Maldacena, "Closed strings from decaying D-branes," arXiv:hep-th/0303139

[9] A. de la Macorra and G. Piccinelli, Phys. Rev. D 61, 123503 (2000) arXiv:hep-ph/9909459.

[10] A. de la Macorra and G. German, Int. J. Mod. Phys. D 13, 1939 (2004).

[11] E. J. Copeland, M. R. Garousi, M. Sami and S. Tsujikawa, Phys. Rev. D 71, 043003 (2005) arXiv:hep-th/0411192.

[12] T. Padmanabhan, "Accelerated expansion of the universe driven by tachyonic matter," Phys. Rev. D 66, 021301 (2002) arXiv:hep-th/0204150; J. S. Bagla, H. K. Jassal and T. Padmanabhan, "Cosmology with tachyon field as dark energy," Phys. Rev. D 67, 063504 (2003) arXiv:astro-ph/0212198. 